\title{
Relation between Heberden's nodes and distal interphalangeal joint osteophytes and their role as markers of generalised disease
}

\author{
Flavia M Cicuttini, Juliet Baker, Deborah J Hart, Tim D Spector
}

\begin{abstract}
Objective-Heberden's nodes are often used as a marker for osteoarthritis (OA). This study examined how often Heberden's nodes and radiological distal interphalangeal (DIP) osteophytes coexist in the same digit and the sensitivity, specificity, and positive predicative value of each for $O A$ at different sites or generalised disease.

Methods-This was a population-based study of 660 middle aged women taking part in a twin study of OA. Distal interphalangeal osteophytes were defined radiologically and graded on a four point scale (0-3) using a published atlas of individual features. Heberden's nodes were defined by standardised clinical examination. OA in other joints (knees, proximal interphalangeal (PIP) joints and carpometacarpal (CMC) joints) was defined radiologically using a published atlas.

Results-Poor agreement was observed between a Heberden's node (HN) and a radiological distal interphalangeal osteophyte in the same finger of the same hand ( s statistic $(95 \% \mathrm{CI})=0.36(0.33,0.39))$. Although HN and radiological DIP osteophytes had similar sensitivity, the specificity and positive predicative value of DIP osteophytes was considerably higher for detecting knee, CMC, PIP OA, and OA in more than two groups of joints (knee, CMC, and DIP joints).
\end{abstract}

Conclusion-HN are not synonymous with DIP osteophytes. Radiological DIP osteophytes are a better marker of knee and multiple joint OA than HN. HN may still be an imperfect surrogate for hand OA when radiology is impractical, but are not an accurate marker of generalised disease.

(Ann Rheum Dis 1998;57:246-248)

The presence of clinically detected Heberden's nodes $(\mathrm{HN})$ is often used as a marker of osteoarthritis (OA). ${ }^{12}$ Minimal information is available on the biological relation between $\mathrm{HN}$ and distal interphalangeal joint (DIP) osteophytes. Most workers have tended to look at one or the other and not both and there has been the underlying assumption that they are one and the same thing. However, the histology of $\mathrm{HN}$ remains unclear and a variety of pathologies have been described. Some workers have described the findings of bony outgrowths associated with classic features of $\mathrm{OA}$ in the cartilage, ${ }^{34}$ while others have observed hypertrophic cartilage. ${ }^{5}$ In other instances, mucoid transformation of the periarticular fibroadipose tissue associated with proliferation of myxoid fibroblasts and cyst formation has been described. ${ }^{6}$

Recently it was suggested that clinical $\mathrm{HN}$ are inferior to radiological examination in predicting knee OA. ${ }^{7}$ We examined the role of $\mathrm{HN}$ and DIP as markers of knee OA and multiple joint involvement in $\mathrm{OA}$ in a population-based study of 660 middle aged women.

\section{Methods}

Participants were taking part in a twin study of OA that has previously been described. ${ }^{8}$ In brief, the study population consisted of female twins aged 48-69 years selected from two sources of volunteers: a normal twin register held in the Institute of Psychiatry, London and directly through an advertising campaign in the media. This population of middle aged women did not differ in prevalence of OA or risk factors for OA (that is, age, weight, smoking, physical activity) from another populationbased sample of middle aged women previously described. ${ }^{9}$ The demographic features of the population were as follows: mean (SD) age 56.4 (6.8) years, mean (SD) weight 63.7 (10.9) $\mathrm{kg} ; 47.2 \%$ past or current smokers; $89 \%$ postmenopausal; and mean (SD) age of menopause 48.9 (6.3) years.

The analyses presented in table 1 refer to the DIP joints (6590) examined as separate units. Hand joints were examined in a systematic way using previously validated and reproducible techniques where anterior swellings were scored after training with an experienced observer. ${ }^{710} \mathrm{HN}$ were classified as: grade $0=$ no bony swelling, grade $1=$ definite bony swelling, not severe; grade $2=$ severe bony swelling but no deformity; grade $3=$ severe bony swelling. Methods for radiographic assessment have previously been described. ${ }^{8}$ DIP
Accepted for publication 19 February 1998 
Table 1 Agreement between Heberden's node and radiological distal interphalangeal osteophytes in the same finger in 6590 digits

\begin{tabular}{|c|c|c|c|}
\hline \multirow[b]{2}{*}{ Heberden's nodes } & \multicolumn{3}{|c|}{ Radiological DIP osteophytes } \\
\hline & Yes & No & Total \\
\hline Yes & 433 & 438 & $871(13.2 \%)$ \\
\hline No & 631 & 5088 & $5719(86.8 \%)$ \\
\hline Total & $1064(16.1 \%)$ & $5526(83.9 \%)$ & 6590 \\
\hline
\end{tabular}

$\kappa$ statistic of agreement between Heberden's nodes (based on clinical grade $\geqslant 1$ ) and radiological osteophytes (based on radiological grade $\geqslant 1)(95 \% \mathrm{CI})=0.36(0.33,0.39)$.

osteophytes were graded on a four point scale (0-3) using a published atlas of individual features. ${ }^{11}$ OA of the knee (knee OA) was defined as having either osteophytes and/or joint space narrowing in either the medial and/or lateral tibiofemoral and/or the patellofemoral compartments of the knee. Classification of proximal interphalangeal (PIP) OA and first carpometacarpal joint of the thumb (1st CMC) was based on a previously validated and similar four point scoring system devised by Kallman et al. ${ }^{12}$

The interobserver and intraobserver reproducibility was assessed on 50 sets of radiographs and 50 hands. The within observer agreement was high for $\mathrm{HN}$ ( $\kappa$ statistic 0.78), DIP osteophyte $(\kappa=0.80)$, and knee OA $(\kappa 0.88)$. The inter-observer agreement between two observers was $\kappa 0.78$ for $\mathrm{HN}, \kappa 0.80$ for DIP osteophytes, and $\kappa 0.88$ for knee OA. These results were comparable to our findings in other similar studies. ${ }^{3-5} \kappa$ Coefficients were also calculated to determine the degree of agreement between radiological grade of DIP osteophytes and the clinical grade of $\mathrm{HN}$.

To assess the association of radiological DIP osteophytes and clinical HN with knee OA and $\mathrm{OA}$ in multiple joints, only one individual of each twin pair was randomly selected. $\mathrm{HN}$ were defined as present if $\mathrm{HN}$ of clinical grade $\geqslant 1$ were present on one or more digits and DIP osteophytes were defined as present if radiological osteophytes grade $\geqslant 1$ were present on one or more digits. Sensitivity, specificity, and positive predictive values of $\mathrm{HN}$ and DIP osteophytes were calculated for knee, CMC, PIP OA and OA involving $\geqslant 2$ groups of joints (two of PIP, CMC or knee). The sensitivity was calculated as the probability of obtaining a positive test, given that it was a true positive. The specificity was calculated as the probability of a test yielding a negative result, given that it was a true negative. The positive predictive value was calculated as the probability of an individual being truly disease positive, given that the test was positive. The $95 \%$ confidence intervals were obtained for the sensitivities, specificities, and positive predictive values by considering them as proportions and applying a normal approximation to the binomial distribution and including a continuity correction. ${ }^{13}$

\section{Results}

Only modest agreement was observed between $\mathrm{HN}$ and radiological DIP osteophytes in the same finger of the same hand $(\kappa(95 \% \mathrm{CI})=$ $0.36(0.33,0.39)$ (table 1$)$. It was found that $16.2 \%$ of DIP joints had DIP osteophytes and $13.2 \%$ clinical $\mathrm{HN}$, and only $6.6 \%$ both. If the $\kappa$ statistic of agreement was recalculated based on a more stringent classification of disease so that grade 1 DIP osteophytes were reclassified as no disease and grade $1 \mathrm{HN}$ were reclassified as no disease, there was still poor agreement $(\kappa$ $=0.27(0.24,0.30))$.

The sensitivity $(95 \% \mathrm{CI})$ and specificity ( $95 \% \mathrm{CI}$ ) of $\mathrm{HN}$ for DIP osteophytes at any digit was $0.41(0.38,0.44)$ and $0.92(0.91$, 0.93) respectively. When a more stringent grading of DIP osteophytes (presence classified as grade $>=2$ ), the sensitivity improved 0.72 $(0.66,0.77)$ with little change in specificity $0.89(0.88,0.90)$. The positive predictive value of $\mathrm{HN}$ for DIP osteophytes was 0.50 (0.46, $0.53)$, increasing to $0.80(0.78,0.82)$ when DIP osteophytes were classified as present using the more stringent grading $(>=2)$.

Radiological DIP osteophytes had generally better specificity and positive predictive value for detecting knee, CMC, PIP OA, and OA in more than two groups of joints (knee, CMC, and DIP joints) than HN (table 2). The sensitivity of radiological DIP osteophytes and $\mathrm{HN}$ for each of the above types of OA was similar.

\section{Discussion}

This study has shown modest agreement between clinical $\mathrm{HN}$ and radiological DIP osteophytes in the same finger. Radiological DIP osteophytes had higher specificity and positive predictive value for detecting knee, CMC, PIP OA, and OA in more than two groups of joints (knee, CMC, and DIP joints) than HN.

"Sensitivity" is the proportion of true positives correctly identified. In this study, the sensitivities for knee OA or multiple joint OA were similar using $\mathrm{HN}$ or DIP osteophytes as markers. "Specificity" is the proportion of negatives correctly identified. Our study showed a negative DIP osteophyte result correctly excluded knee OA $69 \%$ of the time and multiple joint $\mathrm{OA} 81 \%$ of the time. In

Table 2 The sensitivity, specificity, and positive predicative value of Heberden's nodes and distal interphalangeal osteophytes for different forms of osteoarthritis

\begin{tabular}{|c|c|c|c|c|c|c|}
\hline \multirow[b]{2}{*}{ Form of osteoarthritis } & \multicolumn{3}{|l|}{ Heberden's nodes* } & \multicolumn{3}{|c|}{ Distal interphalangeal osteophytes* } \\
\hline & Sensitivity & Specificity & $\begin{array}{l}\text { Positive predictive } \\
\text { value }\end{array}$ & Sensitivity & Specificity & $\begin{array}{l}\text { Positive predictive } \\
\text { value }\end{array}$ \\
\hline TF OA & $0.63(0.51,0.75)$ & $0.48(0.41,0.55)$ & $0.26(0.19,0.32)$ & $0.63(0.51,0.74)$ & $0.74(0.68,0.79)$ & $0.38(0.29,0.47)$ \\
\hline PF OA & $0.56(0.45,0.66)$ & $0.45(0.38,0.51)$ & $0.28(0.21,0.35)$ & $0.47(0.36,0.56)$ & $0.71(0.65,0.76)$ & $0.37(0.28,0.46)$ \\
\hline Knee OA & $0.58(0.47,0.67)$ & $0.47(0.40,0.54)$ & $0.37(0.29,0.44)$ & $0.52(0.42,0.61)$ & $0.75(0.69,0.80)$ & $0.51(0.41,0.60)$ \\
\hline $\mathrm{CMC}$ & $0.69(0.60,0.78)$ & $0.58(0.50,0.65)$ & $0.52(0.43,0.60)$ & $0.60(0.50,0.68)$ & $0.76(0.69,0.82)$ & $0.63(0.53,0.72)$ \\
\hline PIP OA & $0.83(0.73,0.92)$ & $0.57(0.49,0.63)$ & $0.36(0.28,0.44)$ & $0.90(0.82,0.97)$ & $0.77(0.70,0.82)$ & $0.53(0.43,0.63)$ \\
\hline $\mathrm{OA}$ in $\geqslant 2$ joint groups & $0.68(0.58,0.77)$ & $0.520 .45,0.59)$ & $0.41(0.33,0.49)$ & $0.68(0.59,0.77)$ & $0.81(0.76,0.86)$ & $0.61(0.51,0.69)$ \\
\hline
\end{tabular}

$\star$ Point estimate $(95 \% \mathrm{CI})$. 
contrast, for absence of $\mathrm{HN}$, the corresponding results were only $47 \%$ and $52 \%$ correct. The positive predictive value (PPV) is the probability of an individual having multiple joint $\mathrm{OA}$ given that the individual has either DIP osteophytes or HN. The PPV for multiple joint involvement was higher for individuals with DIP osteophytes $(61 \%)$ than for $\mathrm{HN}(41 \%)$. This difference could not be explained by the small difference in prevalence $(16.1 \%$ versus $13.2 \%)$. Overall, these results suggest that DIP osteophytes are a better marker of knee OA and multiple joint $\mathrm{OA}$ that $\mathrm{HN}$.

A previous population-based study using different subjects showed, as with this study, that hand radiology was a better predictor of knee disease than clinical hand examination. ${ }^{7}$ This is consistent with previous studies that showed that HN are not necessarily associated with OA in other joints. ${ }^{2}$ It is possible that different radiographic views might have revealed more radiological signs of OA, but lateral views are difficult to obtain and have largely been abandoned. In our view, it is unlikely to have caused a serious underestimate of osteophytes. Furthermore, using a more stringent radiological grading (radiological DIP OA present if grade $\geqslant 2$ ) did not improve the agreement between $\mathrm{HN}$ and radiological osteophytes $(\kappa=$ $0.27(0.24,0.30))$.

There is a lack of a clear definition of $\mathrm{HN}$ in the literature. In standard rheumatology text books, the terms DIP OA and HN are often used interchangeably. However, the histology of $\mathrm{HN}$ remains unclear and a variety of pathologies have been described, including the presence of bony outgrowths that are consistent with osteophytosis, ${ }^{3}{ }^{4}$ cartilage hypertrophy ${ }^{5}$ or even mucoid transformation of the periarticular fibroadipose tissue associated with proliferation of myxoid fibroblasts and cyst formation. ${ }^{6}$

In conclusion, our results show that DIP osteophytes and $\mathrm{HN}$ are only weakly correlated in the same digit and that as a marker of susceptibility to generalised disease, the presence of DIP osteophytes is a more reliable tool. The poor performance of $\mathrm{HN}$ as a marker of OA elsewhere and our ignorance of the underlying pathology suggests that its role in clinical research and practice needs to be re-evaluated.

1 McCarty DJ, Koopman WJ. Arthritis and allied condition 12th ed. Philadelphia: Lea and Febiger, 1993.

2 Stecher RM. Heberden's nodes. Heredity in hypertrophic arthritis of the finger joints. Am J Med Sci 1941;201:801.

3 Charcot JM. Clinical lectures on senile and chronic diseases. London: T Payne, 1803: 148.

4 Nichols EH, Richardson FL. Arthritis deformans. J Med Res 1909;16:149-221.

5 Fassbender HG. Pathology of rheumatic diseases. New York: Springer-Verlag, 1975.

6 Begg MW, Scott JE. Hyaluronic acid and protein in simple ganglia and Heberden's nodes. Ann Rheum Dis 1966;25: 45-8.

7 Hart DJ, Spector TD, Egger P, Coggon D, Cooper C. Defining osteoarthritis of the hand for epidemiologic studies: The Chingford Study. Ann Rheum Dis 1994;53: $220-3$.

8 Spector TD, Cicuttini F, Baker J, Hart DJ. Genetic influences on osteoarthritis: a twin study BMJ 1996;312: 940-3

9 Hart D J, Spector T D. Cigarette smoking and risk of osteoarthritis in the general population: the Chingford Study. Ann Rheum Dis 1993;52:93-6.

10 Hart DJ, Spector TD, Brown P, Wilson P, Doyle DV, Silman AJ. Clinical signs of early osteoarthritis: reproducibility and relation to x-ray changes in 541 women in the general population. Ann Rheum Dis 1991;50:467-70.

11 Burnett S, Hart DJ, Cooper C, Spector TD. A radiographic atlas of osteoarthritis. London: Springer-Verlag, 1994.

12 Kallman DA, Wigley FM, Scott WW, Hochberg MC, Tobin JD. New radiographic grading scales for osteoarthritis of JD. New radiographic grading scales for oste
the hand. Arthritis Rheum 1989;32:1584-91.

13 Armitage P, Berry G. Statistical methods in medical research. Armitage P, Berry G. Statistical methods in medi 